\title{
Consumo de produtos derivados do tabaco e álcool por estudantes de ciências da saúde
}

\author{
Tobacco products and alcohol consumption \\ among health students
}

Pamella Rossi Minanti ${ }^{1}$, Maria Cristina Cescatto Bobroff ${ }^{2}$

\begin{abstract}
Objetivo: analisar o consumo de produtos derivados do tabaco, narguilé e álcool entre 194 estudantes de cinco cursos da área da saúde, de uma universidade estadual. Método: trata-se de estudo transversal utilizando-se um questionário estruturado pelas autoras e enviado aos estudantes via Google Docs. Resultados: encontrou-se que a maioria $(56,7 \%)$ consome bebidas alcoólicas, 38,65\% fumam narguilé, $12,5 \%$ são fumantes passivos e $4 \%$ fumam cigarro comum. Houve associação significativa entre os fumantes de cigarro e de narguilé $(\mathrm{p}=0,002)$ e entre fumantes de cigarro, de narguilé e a ingestão de bebidas alcoólicas $(\mathrm{p}=0,01)$. Conclusão: esses hábitos não saudáveis precisam ser mais estudados para descobrir suas causas e preveni-las salientando que os participantes da pesquisa serão profissionais da saúde.
\end{abstract}

Palavras-chave: Tabagismo. Alcoolismo. Ocupações em Saúde. Fumar cachimbo de água.

\begin{abstract}
Objective: analyze the consumption of tobacco, hookah and alcohol among 194 health students of five programs from a state university. Method: a cross-sectional study using a structured questionnaire by the authors and sent to students through Google Docs. Results: it was found that the majority $(56,7 \%)$ drink alcoholic beverages, $38,6 \%$ smoke hookah, $12,5 \%$ are passive smokers, and $4 \%$ use common cigarettes. There was a significant association between cigarette smokers and hookah $(p=0,002)$ and between cigarette smokers, hookah smokers and alcohol drinkers $(\mathrm{p}=0,01)$. Conclusion: other researches may be carried on to understand and prevent those unhealthy habits and prevent their causes in the future health professionals.
\end{abstract}

Keywords: Smoking. Alcoholism. Health occupations. Water pipe smoking.

1 Graduação em Enfermagem pela Universidade Estadual de Londrina, Londrina, Paraná, Brasil. Enfermeira no Hospital Evangélico de Londrina, Londrina, Paraná, Brasil. E-mail: pam_minanti@hotmail.com

2 Doutorado em Medicina e Ciências da Saúde pela Universidade Estadual de Londrina, Londrina, Paraná, Brasil. Docente do Departamento de Enfermagem do Centro de Ciências da Saúde da Universidade Estadual de Londrina, Londrina, Paraná, Brasil. 


\section{Introdução}

O tabagismo é um hábito que pode causar e/ ou agravar doenças preexistentes. Estima-se que, a cada ano, milhões de pessoas morrem por doenças relacionadas ao uso de produtos derivados do tabaco no mundo.

A Organização Mundial da Saúde considera que o tabagismo é uma das principais causas de mortes preveníveis mundialmente. Portanto, esforços devem ser mantidos para o controle do uso de produtos derivados do tabaco e para salvar vidas. $^{(1)}$

No Brasil, verifica-se que a prevalência de consumo de cigarros tem diminuído entre os adultos. ${ }^{(2)}$ Nesse aspecto, acredita-se que a existência de políticas públicas vêm obtendo resultados. Apesar disso, grande parte dos jovens e, principalmente, dos adolescentes migraram para o consumo de outras formas de uso de produtos derivados do tabaco. ${ }^{(3)}$ Entre esses novos produtos citam-se o cigarro eletrônico, charuto, cigarrilha, narguilé, entre outros.

Geralmente, entre essas outras formas de tabagismo, a que possui maior prevalência entre os jovens é o narguilé, como na pesquisa do Vigescola com escolares entre 13 e 15 anos, nas cidades de Campo Grande, São Paulo e Vitória;(3) e outra, com estudantes do $3^{\circ}$ e $6^{\circ}$ anos da Faculdade de Medicina da Universidade de São Paulo. ${ }^{(4)}$

Acredita-se que no mundo mais de 100 milhões de pessoas usem o narguilé diariamente, e em alguns países chega a ser mais prevalente que $o$ uso do cigarro. ${ }^{(5)}$ Dados da Pesquisa Especial sobre Tabagismo indicaram que em 2008 havia quase 300 mil consumidores de narguilé no Brasil. ${ }^{(6-7)}$

Também conhecido como cachimbo d'água, water pipe, argileh, goza, hookah ou shisha, é utilizado simultaneamente por várias pessoas e promove o aspecto da socialização entre os jovens.

Vale lembrar, ainda, que a experimentação ou a iniciação nessas novas maneiras de fumar podem ser porta de entrada para o tabagismo regular ${ }^{(8)} \mathrm{e} / \mathrm{ou}$ também causar a dependência nicotínica. ${ }^{(5)}$

Ademais, em revisão sistemática constatou-se uma alta prevalência do consumo de álcool e tabaco entre adolescentes brasileiros. Salienta-se que esses hábitos não saudáveis tendem a se consolidar na vida adulta. ${ }^{(9)}$

Assim, considerando que os alunos da área da saúde serão os profissionais que futuramente atuarão na promoção e prevenção da saúde e de doenças cronicodegenerativas, muitas advindas de hábitos ou estilo de vida não saudáveis, o objetivo desta pesquisa foi analisar a frequência do consumo ativo e passivo de produtos derivados do tabaco, narguilé e álcool entre acadêmicos da área da saúde de uma universidade estadual do estado do Paraná, Brasil.

\section{Material e Método}

Estudo observacional de delineamento transversal. De um total de 1470 acadêmicos matriculados nos cursos de Enfermagem $(n=220)$, Farmácia $(n=260)$, Fisioterapia $(n=226)$, Medicina $(n=482)$ e Odontologia $(n=282)$ do primeiro ao último ano dos cursos da área da saúde de uma universidade estadual pública do Norte do Paraná, 194 concordaram em participar do estudo e fizeram parte da amostra; não houve perdas.

$\mathrm{O}$ instrumento de pesquisa foi construído pelas autoras e estruturado em três partes: a) caracterização sociodemográfica dos participantes; b) hábitos relacionados ao tabagismo passivo ou ativo (cigarro comum e narguilé), e ao uso de bebidas alcoólicas; c) papel do profissional de saúde na prevenção da saúde da coletividade.

A coleta de dados foi realizada de março a maio de 2015 após a autorização prévia do coordenador de colegiado dos cursos e aprovação do Comitê de Ética em Pesquisa da Universidade Estadual de Londrina (CAAE $\mathrm{n}^{\circ}$ 39532214.3.0000.5231, Parecer $n^{\circ} 963.051$ de 05/02/2015).

Os alunos foram contatados pelo endereço eletrônico, no qual foi enviado um link que remetia ao instrumento de pesquisa (via Google Docs). Antes de iniciar a responder, foram informados sobre os objetivos da pesquisa e Termo de 
Consentimento Livre e Esclarecido, concordando assim em participar do estudo.

O banco de dados foi criado em uma planilha do programa Google Docs e, posteriormente, analisado no programa SPSS em frequências e frequências relativas, aplicado o teste exato de Fisher, para associação das variáveis, considerando IC 95\%.

Para manter o sigilo e a confidencialidade em relação aos achados desta pesquisa, os cursos de graduação foram identificados como curso A, B, C, D e E aleatoriamente.

\section{Resultados}

Participaram do estudo 194 universitários, da primeira à última série dos cursos da área da saúde, como demonstrado na Tabela 1.

Tabela 1 - Frequência de acadêmicos e porcentagem de participação dos cursos pertencentes à área da saúde da Universidade Estadual de Londrina, Londrina - PR, 2015.

\begin{tabular}{ccc}
\hline CURSO & FREQUÊNCIA (n) & PORCENTAGEM (\%) \\
\hline D & 58 & $29,90 \%$ \\
A & 57 & $29,40 \%$ \\
B & 35 & $18,00 \%$ \\
E & 24 & $12,40 \%$ \\
C & 20 & $10,30 \%$ \\
\hline TOTAL & $\mathbf{1 9 4}$ & $\mathbf{1 0 0 \%}$ \\
\hline
\end{tabular}

Fonte: Autoras

Caracterização sociodemográfica da amostra

A maioria dos acadêmicos da amostra ( $n=49)$ está cursando a quarta série da graduação. Do total, $73,2 \%$ são do sexo feminino e $26,8 \%$ do sexo masculino. A maioria (67\%) encontrase na faixa etária entre 20 e 25 anos. $\mathrm{O}$ maior percentual de tabagistas também faz parte dessa mesma faixa etária.

A maioria dos estudantes (99\%) é solteira, $62,4 \%$ católicos e $53,6 \%$ ainda moram com os pais, sendo que a renda familiar mensal de três a quatro salários mínimos foi predominante (34\%).

\section{Consumo de produtos derivados do tabaco}

Conforme a Tabela 2, salienta-se que a maioria dos estudantes da amostra $(82,47 \%)$ não é tabagista, sendo que $10,3 \%$ desses são fumantes passivos. Além disso, somente 10 consideraramse fumantes (sendo quatro do curso $\mathrm{D}$, quatro do curso A e dois do curso B).

Tabela 2 - Classificação dos acadêmicos da área da saúde quanto ao hábito de fumar; Universidade Estadual de Londrina, Londrina - PR, 2015.

\begin{tabular}{lcc}
\hline CATEGORIA & FREQUENCIA (n) & PORCENTAGEM (\%) \\
\hline NÃO-FUMANTE & 160 & $82,50 \%$ \\
FUMANTE PASSIVO & 20 & $10,30 \%$ \\
FUMANTE & 08 & $4,10 \%$ \\
EX-FUMANTE E FUMANTE PASSIVO & 04 & $2,10 \%$ \\
EX-FUMANTE & 02 & $1,00 \%$ \\
\hline TOTAL & 194 & $100 \%$ \\
\hline
\end{tabular}

Fonte: Autoras 
A idade de iniciação do uso de produtos do tabaco relatada pela maioria dos tabagistas foi entre 16 e 20 anos. Cerca de $27 \%$ da amostra convivem com tabagistas diariamente, ou seja, são fumantes passivos, sendo que sete desses são também tabagistas.

Questionados sobre o que os induziu ao uso do tabaco, as opções mais respondidas foram: por influência de amigos e pela curiosidade própria (42,9\%). A sensação de bem-estar foi citada por $50 \%$ como o motivo de usar ou ter usado o cigarro.

Entre os 10 acadêmicos que se consideram fumantes, todos já experimentaram cigarro comum com filtro, seis já utilizaram narguilé e cigarro de palha, quatro já experimentaram cigarro sem filtro, cinco já utilizaram charuto, três já usaram cigarro eletrônico e cachimbo e dois já consumiram cigarro comum e narguilé concomitantemente.

Entre os seis acadêmicos que se consideram ex-tabagistas, quatro já experimentaram cigarro comum com filtro, bem como narguilé, e um já utilizou cigarro de palha, como também cigarro sem filtro, cigarro eletrônico, cigarro comum e narguilé no mesmo momento. Nenhum participante indicou o uso de cigarrilha ou de cigarro mascável.

Aqueles que utilizaram o narguilé alguma vez na vida $(47,42 \%)$ destacaram que o motivo do consumo é por estar associado à diversão. Além disso, 38,65\% são consumidores ativos e costumam acrescentar essências de diversos sabores ao fornilho.

Entre os tabagistas (cigarro comum $\mathrm{n}=4 \%$; narguilé $\mathrm{n}=38,65 \%$ ) e ex-tabagistas, metade relatou já ter usado narguilé antes de fumar o cigarro. Entre os 47,42\% que já utilizaram, a relação inversa indica que $n=37,62 \%$ não fumaram cigarro antes do narguilé.

Aplicando-se o teste exato de Fisher constata-se que não houve associação significativa entre o uso de cigarro ou narguilé e o sexo $(p=0,05)$, ou seja, o fator sexo não contribui para a experimentação do narguilé.

\section{Consumo de bebidas alcoólicas}

O consumo de bebidas alcoólicas foi um hábito confirmado por $56,7 \%$ da amostra e mais frequente no sexo feminino (40,72\%). Assim como o tabaco, a frequência de consumo de bebida alcoólica ocorre principalmente aos finais de semana. Da mesma maneira que ocorre com o cigarro, 51,8\% disseram que consomem bebidas alcoólicas devido à sensação de bem-estar.

A aplicação do teste exato de Fisher demonstra que houve associação extremamente significativa entre os fumantes de cigarro e de narguilé $(p=0,002)$. Além disso, verificou-se também associação significativa entre o uso de cigarro comum com filtro, o uso de narguilé e a ingestão de bebidas alcoólicas $(p=0,01)$. No entanto, não houve associação entre o sexo e uso de bebida alcoólica $(\mathrm{p}=0,371)$.

\section{Discussão}

Os resultados obtidos com este estudo são relevantes, já que o maior grupo de acadêmicos se diz não fumante. Isso reforça a hipótese de que universitários das Ciências da Saúde estão conscientes sobre seus malefícios devido ao aprendizado adquirido durante a graduação, apesar de ser um tema que ainda merece pesquisas mais aprofundadas e de maior abrangência. Por outro lado, 4\% ainda sustentam hábitos de vida não saudáveis neste aspecto. Ademais, aqueles que consomem o narguilé não se consideram tabagistas. Portanto, será que o narguilé não é considerado hábito tabágico pelos jovens por ser associado à diversão ou ainda há desconhecimento sobre os malefícios do mesmo?

Apesar de a maior concentração de acadêmicos serem do quarto ano, os resultados não demonstraram associação entre a série da graduação e o uso do tabaco. Portanto, não se pode afirmar o mesmo que outros pesquisadores sobre o consumo de substâncias psicoativas (entre elas o tabaco) por estudantes de medicina, que 
aumentou da primeira à última série. ${ }^{(10)}$

Embora vários estudos indiquem uma prevalência relativamente maior de tabagistas no sexo masculino ${ }^{(3,11-12)}$ tal fato não ocorre na presente pesquisa. Pesquisadores do estado do Paraná detectaram que mulheres casadas e também aquelas com escolaridade de nível superior são menos adeptas ao hábito de fumar. ${ }^{(13)}$ No entanto, tais considerações podem servir de alerta às mulheres modernas tendo em vista a mudança de hábitos deste grupo que tem fumado tanto quanto os homens, ${ }^{(11)}$ o que pode alterar epidemiologicamente o processo saúde-doença daqui a alguns anos com o aumento da incidência de doenças pulmonares e o agravamento de outras doenças causadas pelo tabagismo.

A maioria dos acadêmicos tem alguma crença religiosa, embora não tenha havido associação entre essas variáveis. A prática religiosa semanal mostrou-se como um fator protetor em relação ao consumo de álcool entre universitários da área da saúde. ${ }^{(14)}$

Quanto à moradia, esperava-se que o consumo de álcool e tabaco estivesse relacionado àqueles que moram fora da casa dos pais (sozinhos, em república, com amigos, entre outros), da mesma maneira que o encontrado em estudo da Universidade Federal de Pelotas, que afirma um maior percentual de tabagistas entre os que moram sozinhos e consumidores de bebidas alcoólicas entre os que moram com amigos. ${ }^{(12)}$ No entanto, a maioria dos universitários desta pesquisa, moradores em república, consomem bebidas alcoólicas.

O início do uso de drogas psicoativas está cada vez mais precoce, principalmente em relação ao álcool e tabaco. Em Salvador, constatou-se que mais da metade dos escolares experimentou o primeiro cigarro antes dos $15 \operatorname{anos}^{(15)} \mathrm{e}$, mais recentemente, a faixa etária para a primeira experimentação por tabagistas estudantes da área da saúde foi de 11 a 23 anos. $^{(11)}$

Corroborando a literatura encontrada, os estudantes deste estudo relataram início do uso de produtos derivados do tabaco quando adolescentes (mínimo de 10 e máximo de 20 anos). Uma das explicações para isso é que na adolescência os jovens procuram por liberdade, sendo esta a fase de busca pela independência, tornando-se também vulneráveis à experimentação das drogas. ${ }^{(16)}$

Acredita-se que, entre vários fatores, o hábito de fumar seja influenciado também pela convivência com tabagistas próximos. Pesquisa em duas universidades brasileiras demonstrou que ter amigos, irmãos, namorado ou companheiro fumante aumenta em seis vezes o risco desse hábito, ${ }^{(17)}$ talvez pela facilidade de acesso ao produto no domicílio. Neste estudo, entre os 14 usuários de produtos derivados do tabaco, 10 convivem com tabagistas frequentemente, o que corresponde a $71,4 \%$ aproximadamente.

Apesar de existir no Brasil lei que proíbe fumar em ambientes coletivos fechados a proteger a população da exposição passiva, vale lembrar que fumantes passivos também devem ficar atentos. As substâncias exaladas pelo fumo são cumulativas no organismo e os efeitos se intensificam conforme o tempo de exposição à fumaça. ${ }^{(6-7)}$ Apesar de haver poucos tabagistas entre os cursos da área da saúde, o número de fumantes passivos encontrado na atual pesquisa é considerado elevado (12,37\%).

Existem múltiplos fatores associados à iniciação do uso de tabaco, entre os encontrados na literatura estão: idade entre 15 e 19 anos, tabagismo de pessoas próximas (pais, irmãos, amigos ou namorado), uso de álcool, influência da mídia, pais separados, correção agressiva ou coerciva dos pais, falta de instrução sobre drogas em casa, ${ }^{(15)}$ aumento da idade, baixa escolaridade dos pais, possuir renda própria, lares monoparentais ou sem os pais e percepção dos filhos de que os pais não se importariam se fumassem. ${ }^{(8)}$ Tabagismo de pessoas próximas e idade maior do que 15 anos para a experimentação são duas variáveis associadas ao tabaco identificadas neste estudo.

Produtos derivados do tabaco costumam proporcionar a socialização entre os jovens, o mesmo se aplica ao álcool. Geralmente, ambos estão associados à diversão, sensação de bem- estar, liberdade, relaxamento e poder. Entre estudantes de 
medicina, a diversão e o relaxamento são citados como as principais razões para manter o uso de substâncias psicoativas; ${ }^{(10)}$ estudantes da área da saúde também citaram a quebra da rotina, curtição dos efeitos da droga, redução da ansiedade/estresse e participação em grupos de amigos. ${ }^{(16)}$

Os acadêmicos desta pesquisa relataram terem sido motivados à experimentação do tabaco por influência de amigos e pela própria curiosidade. Tal resultado também pôde ser encontrado em outros estudos realizados tanto com universitários como com escolares. ${ }^{(11,15)}$

Os resultados são significativos em relação ao uso doálcool, já que mais da metade dos universitários consome bebidas alcoólicas frequentemente e em maior concentração no sexo feminino, talvez devido ao predomínio do mesmo na amostra. Entretanto, alguns trabalhos constatam um maior consumo de bebidas alcoólicas pelos homens. ${ }^{(18-19)}$

Outra pesquisa brasileira, com universitários da área da saúde, constatou a prevalência de consumo de álcool elevada no sexo masculino (85\%); embora o perfil do consumo ser de baixo risco $(77,1 \%)$, a exposição ao alcoolismo é precoce entre os futuros profissionais. Esses autores também constataram que o consumo elevado de álcool entre os jovens acadêmicos é semelhante aos resultados de outros estudos brasileiros e internacionais. ${ }^{(14)}$

O álcool é a substância psicotrópica mais consumida pelos jovens no Brasil e no mundo. ${ }^{(20)}$ Comparado a outros estudos, verificou-se que o consumo de álcool entre estudantes da área da saúde tem sido elevado. Pesquisas mais recentes encontradas mostram que $27,1 \%$ de etilistas entre 243 estudantes de Enfermagem e, entre esses, $42,3 \%$ consomem álcool de uma a cinco vezes por semana, considerados como consumidores de bebida alcoólica entre moderados a graves ${ }^{(21)}$ de acordo com critérios do Ministério da Saúde do Brasil utilizados naquele estudo.

Houve associação entre o consumo de tabaco e álcool considerando-se que todos os fumantes são etilistas. $\mathrm{O}$ mesmo ocorreu em pesquisa com estudantes de odontologia na Paraíba. (22) Em relação ao narguilé e álcool, $75 \%$ dos narguileiros desta pesquisa são também etilistas.

O consumo de tabaco é ainda maior quando associado ao álcool, como mostra o estudo com universitários tabagistas da Faculdade de Minas Gerais, no qual $67 \%$ afirmaram fumar mais quando consomem bebida alcoólica. ${ }^{(23)}$ Entre estudantes da área da saúde da Universidade Estadual do Oeste do Paraná, de Cascavel, 82\% também afirmam que a ingestão de álcool estimula o tabagismo. ${ }^{(11)}$ Além disso, salienta-se que essa concomitância aumenta o risco para câncer oral, de laringe e de esôfago, entre outros.

Explica-se que, com a ingestão de álcool a nicotina se dissolve mais facilmente na corrente sanguínea, fazendo com que uma menor quantidade da mesma chegue ao sistema nervoso. Ao beber, a substância é excretada pelos rins mais rapidamente, pois o álcool é inibidor do hormônio antidiurético. Com isso, a queda da concentração da droga no sangue desencadeia sintomas de abstinência e, consequentemente, um maior desejo de fumar. ${ }^{(24)}$

Pesquisa recente ressalta que o uso de narguilé entre universitários americanos é um problema de saúde atual e que merece atenção especial. Demonstrou-se alta prevalência de usuários, entre os participantes, em consonância com outros estudos citados no mesmo artigo, além de que os estudantes têm pouca percepção do potencial aditivo do narguilé, ${ }^{(25)}$ o que corrobora para a preocupação com hábitos de saúde dessa população jovem.

Apesar das limitações desta pesquisa (amostra de uma universidade e alunos de uma área específica: saúde) constatam-se importantes resultados. Nesse aspecto, pesquisas adicionais são necessárias para aprofundar o conhecimento nessa área.

\section{Conclusão}

Conclui-se que o hábito de experimentar o narguilé é comum entre os estudantes da área da saúde.

Considerado um importante problema de Saúde Pública, não existe forma segura de consumo de produtos derivados do tabaco, devendo ser mundialmente combatido, principalmente entre os 
estudantes da área da saúde, por serem os futuros responsáveis pela difusão de orientações básicas à comunidade. Apesar de o governo ter incluído políticas públicas para o combate ao tabagismo a indústria produtora ainda insiste em estimular o consumo. Importa investir na prevenção por meio de abordagens educacionais direcionadas a estudantes universitários com esclarecimentos sobre os riscos à saúde.

A associação significativa entre o uso de cigarro comum, o uso de narguilé e a ingestão de bebidas alcoólicas reforça a necessidade de novos estudos com outras abordagens metodológicas. Ademais, a adesão de adolescentes e adultos jovens a esses hábitos e a aceitação da sociedade, e principalmente dos próprios familiares, contribuem para agravar ainda mais tal situação.

\section{Referências}

1 World Health Organization. WHO report on the global tobacco epidemic, 2015: raising taxes on tobacco [Internet]. 2015 [cited 2015 Nov 1]. Available from: http://apps.who.int/iris/ bitstream/10665/178574/1/9789240694606 eng.pdf?ua $=1 \& u a=1$

2 Godoy I. Prevalência de tabagismo no Brasil: medidas adicionais para o controle da doença devem ser priorizadas no Ano do Pulmão. J Bras Pneumol. 2010;36(1):4-5.

3 Szklo AS, Sampaio MMA, Fernandes EM, Almeida LM. Perfil de consumo de outros produtos de tabaco fumado entre estudantes de três cidades brasileiras: há motivo de preocupação? Cad Saúde Pública [Internet]. 2011 [citado 2018 maio 20];27(11):2271-5. Disponível em: http://www.scielo.br/pdf/csp/ v27n11/20.pdf

4 Martins SR, Paceli RB, Bussacos MA, Fernandes FLA, Prado GF, Lombardi EMS, et al. Experimentação de e conhecimento sobre narguilé entre estudantes de medicina de uma importante universidade do Brasil. J Bras Pneumol. 2014;40(2):102-10.
5 Viegas CAA. Formas não habituais do uso do tabaco. J Bras Pneumol. 2008;34(12):1069-73.

6 Instituto Nacional de Câncer. Crescimento do uso do narguilé no Brasil preocupa. Rede Câncer [Internet]. 2012 [citado 2018 maio 20];19(3):36-8. Disponível em: https:// www.inca.gov.br/sites/ufu.sti.inca.local/ files//media/document//epidemiologia-redecancer-19.pdf

7 Instituto Nacional de Câncer. INCA alerta para malefícios do narguilé [Internet]. 2012 [citado 2018 maio 20]. Disponível em: http://www.blog.saude.gov.br/promocao-dasaude/30976-inca-alerta-para-maleficios-doconsumo-do-narguile

8 Barreto SMM, Giatti L, Oliveira-Campos M, Andreazzi MA, Malta DC. Experimentation and use of cigarette and other tobacco products among adolescents in the Brazilian state capitals (PeNSE 2012). Rev Bras Epidemiol [Internet]. 2014 [cited 2016 Feb 5];17(Suppl 1):62-76. Available from: http:// www.scielo.br/pdf/rbepid/v17s1/1415-790Xrbepid-17-s1-00062.pdf. doi: http://dx.doi. org/10.1590/1809-4503201400050006

9 Barbosa Filho VC, Campos W, Lopes AS. Prevalência de consumo de álcool e tabaco entre adolescentes brasileiros: revisão sistemática. Rev Saúde Públ. 2012;46(5):901-17.

10 Lemos KM, Neves NMBC, Kuwano AY, Tedesqui G, Bitencourt AGV, Neves FBCS, et al. Uso de substâncias psicoativas entre estudantes de medicina de Salvador (BA). Rev Psiq Clín. 2007;34(3):118-24.

11 Silva CCB, Vieira L, Tavares KO, Rodrigues J. Análise descritiva dos estudantes tabagistas dos cursos da área da saúde da Universidade Estadual do Oeste do Paraná. In: Anais do $5^{\circ}$ Congresso Paranaense de Fisioterapia; 2011.

12 Ramis TR, Mielke GI, Habeyche EC, Oliz MM, Hallal PC. Tabagismo e consumo de álcool em estudantes universitários: prevalência e fatores associados. Rev Bras Epidemiol. 2012; 15(2):376-85. 
13 Scarinci IC, Bittencourt L, Person S, Cruz RC, Moyses ST. Prevalence of tobacco use and associated factors among women in Parana State, Brazil. Cad Saúde Pública. 2012;28(8):1450-8.

14 Pelicioli M, Barelli C, Gonçalves CBC, Hahn SR, Scherer JI. Perfil do consumo de álcool e prática do beber pesado episódico entre universitários brasileiros da área da saúde. J Bras Psiquiatr [Internet]. 2017 [cited 2018 June 1];66(3):150-6. Doi: http://dx.doi. org/10.1590/0047-2085000000164

15 Machado Neto AS, Andrade TM, Napoli C, Abdon LCSL, Garcia MR, Bastos FI. Determinantes da experimentação do cigarro e do início precoce do tabagismo entre adolescentes escolares em Salvador (BA). J Bras Pneumol. 2010;36(6):674-82.

16 Chiapetti N, Serbena CA. Uso de álcool, tabaco e drogas por estudantes da área de saúde de uma universidade de Curitiba. Psicol Reflex Crit. 2007;20(2):303-13.

17 Instituto Nacional de Câncer. Pesquisa: vigilância do tabagismo em universitários da área da saúde [Internet]. 2009 [citado 2018 maio 6]. Disponível em: http://www.inca.gov. $\mathrm{br} / \mathrm{wps} / \mathrm{wcm} / \mathrm{connect/agencianoticias/site/}$ home/noticias/2009/pesquisa vigilancia_do_ tabagismo_em_universitarios

18 Faria YO, Gandolfi L, Moura LBA. Prevalence of risk behaviors in young university students. Acta Paul Enferm [Internet]. 2014 [cited 2018 May 25];27(6):591-5. Available from: http:// www.scielo.br/pdf/ape/v27n6/en_1982-0194ape-027-006-0591.pdf

19 Silva ÉC, Tucci AM. Estudo transversal sobre o uso de risco de álcool em uma amostra de estudantes de uma universidade federal brasileira. J Bras Psiquiatr. 2014; 63(4): 317-25.

20 Faria Filho EA. Profile of alcohol and drug use in adolescent pupils in Brazilian tate capital. SMAD: Rev Eletrônica Saúde Mental Alcool Drog [Internet]. 2014 [cited 2015 Oct 24];10(2):78-84. Available from: http://pepsic. bvsalud.org/pdf/smad/v10n2/en_05.pdf
21 Alves EF. Estilo de vida de estudantes de graduação em enfermagem de uma instituição do sul do Brasil. Rev CPAQV. 2011;3(1).

22 Granville-Garcia AF, Branco ACL, Sarmento DJS, Cavalcanti AL, D'Avila S, Menezes VA. Tabagismoefatoresassociadosentreacadêmicos de odontologia. RFO [Internet]. 2009 [citado 2015 out 24];14(2):92-8. Disponível em: http:// files.bvs.br/upload/S/1413-4012/2009/v14n2/ a92-98.pdf

23 Rodrigues Júnior JC, Ferraz SMR, Bruno RX. Prevalência e perfil de tabagistas universitários ingressantes de uma instituição de ensino superior. Pulmão RJ. 2009;18(1):14-8.

24 Rosemberg J. Nicotina: droga universal [Internet]. 2003 [citado 2015 jun 21]. Disponível em: ftp://ftp.cve.saude.sp.gov.br/ doc_tec/cronicas/nicotina.pdf

25 Fevrier B, Nabors L, Vidourek RA, King KA. Hookah use among College students: recent use, knowledge of health risks, attitude and reasons for use [Internet]. J Community Health. 2018 [cited 2018 May 25];4(1):17. Available from: https://doi.org/10.1007/ s10900-018-0519-8
Recebido em: 22 mar. 2019

Aceito em: 24 jan. 2020 